\title{
Management issues in HIV-associated neurocognitive disorders
}

\author{
This article was published in the following Dove Press journal: \\ Neurobehavioral HIV Medicine \\ 10 July 2012 \\ Number of times this article has been viewed
}

\author{
Lucette A Cysique ${ }^{1,3,4}$ \\ Margaret P Bain' \\ Tammy A Lane ${ }^{2}$ \\ Bruce J Brew ${ }^{1,4}$
}

'Department of Neurology, St Vincent's Hospital, Darlinghurst, NSW, Australia; ${ }^{2}$ AIDS Dementia and HIV Psychiatry Service (ADAHPTS) Sydney Hospital and Sydney Eye Hospital, Sydney, Australia; ${ }^{3} \mathrm{St}$ Vincent's Hospital Clinical School, Faculty of Medicine, The University of New South Wales, Sydney, Australia; ${ }^{4}$ St Vincent's Applied Medical Research Centre, Sydney, Australia
Correspondence: Lucette A Cysique Department of Neurology, Xavier Building, Level 4, St Vincent's Hospital, 390 Victoria Street, Darlinghurst, Sydney, NSW 2010, Australia

Tel +6I 283824104

Fax +6I 283824101

Email Icysique@unsw.edu.au
Abstract: The incidence of human immunodeficiency virus (HIV)-associated neurocognitive disorder (HAND) decreased with the introduction of combined antiretroviral therapy (cART), but there is now evidence that HAND is persisting and developing despite effective HIV suppression and the absence of other confounds. HAND in the cART era is different. The main issues are: (1) HIV disease is now a chronic disorder spanning decades. While acute and subacute presentations of HAND still occur, there is mounting evidence that it is based on cumulative brain damage. Furthermore, the pathogenetic significance of continuing HIV replication as the main factor in such brain damage is still unclear. (2) As HIV disease is a chronic disorder with increased life expectancy, the effects of aging are now becoming important. Their impact and significance on the pathogenesis of HAND are only beginning to be grasped. (3) In addition to aging, there is the increased risk of the effects of comorbid conditions (eg, cardiovascular and kidney diseases). The optimal treatment of HAND in the context of cART has not yet been established with any certainty. As a general principle, systemic HIV disease must be well controlled. There is increasing evidence that central nervous system disease should also be well controlled and may require specific antiretroviral drugs that have the ability to effectively penetrate the central nervous system. The role of adjunctive therapies remains hypothetical. Research to date has shown that all adjunctive therapies have thus far failed to show any significant benefit. In this paper, we provide an updated review of the clinical presentation and neuropsychological profile in the cART era. We also review the current HAND diagnostic nomenclature in respect to both its recommendations and limitations. This is followed by discussion of the main management issues for persisting HAND, including screening algorithms and optimal therapeutic options. Finally, we explore the use of everyday life assessment methods and recommendations.

Keywords: HIV/AIDS, antiretroviral treatment, neurological complications, neuropsychological functions, HIV-associated dementia, aging

\section{Introduction}

\section{HIV-associated neurocognitive disorder modified clinical profile in the cART era}

At presentation, most human immunodeficiency virus-positive (HIV+) individuals affected with mild HIV-associated neurocognitive disorder (HAND), now the most common form of the disorder, do not show obvious signs of cognitive disturbance. It is only with careful, focused clinical evaluation and neuropsychological assessment that difficulties are reliably detected. These difficulties typically involve the domains of working memory, speed of information processing, attention, and active retrieval. ${ }^{1}$ 
Moreover, consistent with observations in our clinical experience, a comprehensive assessment of cognitive complaints often reveals that the patient has difficulties in the most demanding activities of everyday life (eg, work, multitasking, planning, organization, and motivation). In some patients it is only when thoroughly questioned that they confess those difficulties that they tend otherwise not to report spontaneously or sometimes to minimize. In moderate to severe forms of HAND, slowness and apathy are much more striking and the impact on everyday life is often evident (eg, job loss, inability to concentrate for a significant period of time, severe working memory and memory difficulties). At this stage patients have variable insight into their difficulties; ${ }^{2}$ therefore, reliance on self-report of cognitive complaints should be viewed with caution. When demented, HIV+ individuals often show disinhibited behavior, severe apathy, slowness, and obvious motor dysfunction (eg, sloppy handwriting, tremor, and poor balance) as well as mild to moderate anosognosia. ${ }^{2}$ Memory function, even at the HIV-associated dementia (HAD) stage, is typically impaired at the level of active retrieval, while recognition is relatively well preserved $^{3}$; this is in contrast with cortical dementias such as Alzheimer's disease (AD). This profile is still the most predominant in the combined antiretroviral therapy (cART) era, with the exception of those instances where combined HAND and AD occur. ${ }^{4}$

Although the advent of cART has rendered severe forms of cognitive deterioration less common, mild to moderate degrees of cognitive disturbances have become more prevalent. ${ }^{5}$ Due to the chronicity of the condition some have hypothesized that the nature and phenotype of cognitive deficits may have changed over the pre- and post-cART eras. ${ }^{1}$ Evidence for this change in the neuropsychological profile is emerging in that learning and mental flexibility difficulties are more prevalent in chronically HIV+ individuals than previously observed, while fine motor-coordination difficulties are somewhat less predominant. ${ }^{1,6}$ This means that screening instruments based only on motor-coordination dysfunction and that do not adequately assess mental flexibility (one of the components of executive functioning) will not have sufficient sensitivity to reliably detect HAND. ${ }^{?}$

Whether these mild changes are significant enough to impact on the individual's everyday life is often questioned. However, cumulative evidence from the neuropsychology literature indicates that such changes can significantly impact on one's independence in daily activities (employment and efficiency at work), ${ }^{8}$ and perhaps most importantly, medication adherence. ${ }^{9,10}$ Even in its mild form, HAND has been shown to be independently predictive of death. ${ }^{11}$
Research also suggests that suffering even a mild episode of HAND has been associated with greater likelihood of having another episode in the future. ${ }^{12}$

\section{Persisting high prevalence and potentially increasing incidence}

While HAND does not affect all individuals diagnosed with HIV, mild neurocognitive impairment is present in approximately $30 \%$ of individuals on cART with asymptomatic HIV infection, without leading to self-perceived everyday repercussions in everyday activities. ${ }^{13}$ However, in individuals with AIDS the prevalence of HAND is up to $50 \%,{ }^{1,14,15}$ as shown in several large international cART cohorts. Such findings indicate that prevalence is steady when compared to the pre-cART era. In those with systemic viral suppression up to $30 \%$ of HIV+ individuals have HAND, ${ }^{13,16}$ with the nondemented forms of HAND accounting for the majority of these cases. Instances of HAD have been found to be relatively rare with prevalence rates falling between $3 \%$ and $8 \%$. In the initial years of the cART era annual HAD incidence was estimated at about $3 \%$ as opposed to about $7 \%$ before cART. ${ }^{5}$ Thus, when including milder cases of HAND, there is now evidence that incidence for HAND is on the rise. For example, the AIDS Longitudinal Linked Randomized Trials (ALLRT) assessed the prevalence and incidence of neurocognitive impairment in $\mathrm{HIV}+$ individuals who initiated cART and found that $39 \%$ of participants were impaired at baseline, $22 \%$ remained impaired on the study, and $21 \%$ became impaired on the study despite optimal medical management $(n=1160) \cdot{ }^{17}$ Lastly, two recent European studies have found rates of neurocognitive impairment in virally controlled HIV+ individuals, which differed from the majority of the neuropsychological studies in the cART era. One study found that $19 \%{ }^{18}$ were impaired and the other one found that $64 \%-84 \%{ }^{19}$ were impaired. On closer inspection, both studies had suboptimal normative data for the sample they studied and/or used nonstandard neuropsychological testing or tests that are known to be less sensitive to HIVrelated impairement. ${ }^{18,19}$ This reinforces the need to use appropriate testing and normative methods, as emphasized in the new HAND criteria nomenclature. ${ }^{20}$

\section{HAND American Academy of Neurology (AAN) nomenclature (2007)}

The current definitional criteria ${ }^{21}$ propose three severity categories for HAND: HIV-associated asymptomatic neurocognitive impairment (ANI), HIV-associated mild neurocognitive disorder (MND), and HAD. This 
three-severity classification system was recently proposed to distinguish better between mild forms of HAND and dementia.

ANI is diagnosed when a patient exhibits neuropsychological performance that is at least one standard deviation (SD) below the mean of demographically adjusted normative scores in at least two cognitive domains. The diagnostic algorithm recommends the assessment of several cognitive domains including attention, working memory, speed of information processing, language, abstraction, executive functioning, complex perceptual motor skills, learning/ memory, simple motor skills, and perceptual abilities. Further, in the case of ANI, the impairment must not occur solely as part of delirium and not be explained by comorbid conditions, such as a previous head injury or current psychiatric disorder. These deficits should not interfere with the individual's everyday functioning.

MND refers to an acquired mild to moderate neuropsychological impairment in cognitive function that is at least one SD below the mean of demographically adjusted normative scores on two measures of distinct cognitive domains. The individual must not meet the criteria for delirium or dementia, and impairments not be explained by comorbid conditions. The distinguishing feature between ANI and MND is that the impairments in MND must interfere, at least mildly, with instrumental activities of daily living (IADL).

In contrast to ANI and MND, HAD is defined by moderate to severe cognitive impairment evidenced by a score that is at least two SDs below demographically adjusted norms in at least two cognitive domains. The impairment must result in a marked difficulty executing IADL, not meet the criteria for delirium, and not be adequately explained by comorbid conditions. HAD is the most severe form of HAND, often rendering the individual incapable not only of employment but independent living.

There are three main points to note in these criteria:

- These diagnostic criteria are centered on the standardized assessment of neuropsychological functions. This is a substantial modification from the original criteria proposed in 1991 and 1996 by the AAN, which recognized apathy and emotional lability as being features of minor motor cognitive disorder and HIV-1-associated dementia. $^{21-23}$ This means that the current criteria render access to standard neuropsychological assessment essential for diagnosis. It should be noted that this is not an isolated situation and is also true for AD. ${ }^{24}$ However, in the face of the milder clinical presentation of HAND, the assessment of a minimum number of cognitive domains (at least five in the current diagnostic criteria ${ }^{20}$ ) is indeed necessary to reliably detect impairment. Because access to a neuropsychologist is not always possible the plausibility of screening patients to efficiently detect HAND should be determined.

- The other major clinical consequence of these criteria is the assessment of IADL to distinguish between ANI and MND. However, how to obtain evidence of IADL decline has not been well described in these new criteria. We would recommend the use of quantitative IADL questionnaires, rather than the use of a series of qualitative questions, for better specificity and standardization. Existing standardized quantitative questionnaires (eg, Patient's Assessment of Own Functioning ${ }^{25}$ ), are typically familiar only to neuropsychologists and are often seen as too lengthy for routine use in HIV clinics; therefore, improvement in this area is needed. It should also be taken into consideration that increased complaints on these questionnaires are more robustly associated with depressive complaints rather than objective impairment on cognitive tests. ${ }^{26} \mathrm{We}$ will discuss further how to overcome this challenge in the section below, which focuses on the patient's perspective.

- Lastly, neuropsychiatric disturbances such as apathy are likely also to be an expression of the disease. Thus, the downgrading of these symptoms from the diagnostic criteria may lead to reductions in sensitivity. Indeed, progression of the disease that is not cognitive but neuropsychiatric is a possibility, as was recognized in the initial AAN criteria. Some of the neuropsychiatric expression of HAND is likely to be due to the underlying striatofrontal pathology, and especially in aging and chronic HIV+ patients, ${ }^{27}$ but there is a lack of research on these issues. At the patient-management level, the clinician should be aware that some neuropsychiatric conditions are likely to worsen HAND clinical presentation. As observed in our clinical practice, this includes such behaviors as high levels of apathy, but also sometimes behavioral disinhibition, poor insight into difficulties, anxiety without obvious clinical depression, poor decision-making in everyday life such as poor financial management, and sometimes impulsivity. Such behavior may not be captured quantitatively, as these patients are usually excluded from research studies. In terms of emerging research into the social and neuropsychiatric consequences of HAND, there are studies that have investigated the facial emotional processing of chronic HIV+ individuals versus demographically comparable controls. Two studies ${ }^{28,29}$ have found mildly 
impaired processing for negative expressions. However, this research needs further confirmation, particularly in terms of long-term prognosis.

Some have challenged the concept of ANI as representing a "misdiagnosis"; that is, a category that has no clinical or neuropathological relevance. ${ }^{30}$ This can be directly challenged by the fact that these criteria have been demonstrated as having both clinical and neuropathological relevance. ${ }^{31}$ Additionally, both recent ${ }^{32}$ and past data ${ }^{12,33}$ have demonstrated that ANI and mild forms of HAND are associated with greater risk of neurocognitive decline. Moreover, this argument is based on an erroneous understanding of psychometric rules underlying neuropsychological-based diagnostic criteria development. More specifically, Gisslen et $\mathrm{al}^{30}$ based their argument on an erroneous appreciation of the underlying statistical recommendation of the HAND criteria because they did not consider the relevant normative data on which the criteria are based..$^{34}$ It is possible that the misunderstanding stems from the HAND nomenclature, as the psychometric requirements for the composition of each cognitive domain were not outlined. Indeed, it must be understood that the outcome of importance behind a cutoff development is the individual neuropsychological measure and not the cognitive domain. To put this into the context of the HAND criteria, the normative work under which they were designed involves test batteries composed of approximately 15-20 individual neuropsychological measures. In other words, the $15 \%$ of "impairment" expected in the normal population is only plausible when such a battery size is used. If fewer individual neuropsychological measures are used, the cutoffs should be less stringent. This binomial distribution rule was very well illustrated by Ingraham and Aiken in 1996, ${ }^{35}$ and then further validated for the Halstead-Reitan Neuropsychological Test Battery $^{35}$ (the battery normed by Heaton et $\mathrm{al}^{34}$ ). This work shows clearly that the expected rate of neurocognitive impairment in a neurologically normal sample decreases as the number of individual neuropsychological measures included in a test battery also decreases. It also discusses what is expected with different levels of between-measures correlation. It should be understood here that those different levels of between-measures correlation "mimic" the effect of disease severity. Therefore, when using the HAND nomenclature guidelines recommendations, and if using a relatively small battery of neuropsychological measures $(<10)$, it is essential that the researcher or clinician revise the expected rate of neurocognitive impairment in their control sample and reformulate their cutoffs. Before use, the new cutoff should ideally be cross-validated in an independent control sample and a clinical sample. The cross-validation in a clinical sample with varying levels of neurocognitive impairment will empirically test different degrees of disease severity and therefore provide a realistic scenario for the level of between-measures correlation. Given the above points, we believe that the rigid reference to $15 \%$ of individuals being classified as impaired in the general population in Gisslen et $\mathrm{al}^{30}$ is erroneous. We would recommend that this point be clearly addressed in a future formulation of the HAND diagnostic criteria.

While the diagnostic criteria for HAND indicate the need to exclude other comorbid conditions that might reasonably account for cognitive impairment, the impact of comorbid conditions such as drug and alcohol use or other medical conditions can be difficult to distinguish in clinical practice, and indeed comorbid factors can have a synergistic effect in HAND. We encourage the reader to refer to other parts of this special issue regarding consideration of the criteria in the context of comorbid conditions, and how HIV-related neurocognitive impairment in general should be interpreted in the context of comorbid neuropsychological confounds (such as in psychiatric, neurological, and some systemic conditions), as they may worsen or compound the HAND profile.

In the cART era, there is also a need to adopt a long-term therapeutic framework as standard practice. New quantitative criteria are needed to address the central question of the clinical significance of cognitive change. In other words, the new diagnostic guidelines need to provide clinically relevant cutoffs for cognitive change (for a review on this issue, see Cysique and $\mathrm{Brew}^{36}$ ) that the clinicians can use to devise an individualized long-term therapeutic plan. The time frame for follow-up testing should be determined depending on the magnitude of the cognitive decline, stability, or improvement.

\section{Main management issues and their optimal therapeutic strategy for persisting HAND Patients who have had previous HAND episodes and past CNS opportunistic infections}

Because the prevalence of HAND has been steady between the pre-cART and cART eras and more patients now survive, it is possible that a "legacy effect" explains the persisting high prevalence of HAND. Supporting this are findings from long-term observational studies that have shown that HAND is more likely in patients who have had previous 
HIV-related central nervous system (CNS) disorders (ie, past HAND and opportunistic infections in the CNS). ${ }^{12,33}$ However, closer inspection reveals that these are only partial effects. Moreover, cumulative evidence suggests that HAND happens de novo despite cART. ${ }^{17}$ At the patient level, this means that extra caution and more regular screening should be considered in patients with past HIV-related CNS diseases.

\section{What is the major cause of the persistence of HAND, and which biomarkers should be investigated?}

HAND can occur in the context of viral suppression. Hence, current CD4-T cell count is not a reliable marker for HAND. In essence, this means that immune reconstitution inflammatory syndrome (IRIS) is unlikely to be a major cause for the persistence of HAND. In contrast, research shows that the nadir CD4 T-cell count is a new risk factor for HAND in the cART era. ${ }^{111,37,38}$ That is, past immune suppression and current neurocognitive status are becoming increasingly associated. For patient management, this means a focus on viral suppression and current CD4 levels as the only biomarkers of reference for HAND is inappropriate. Furthermore, HAND can also occur despite suppression of CSF HIV RNA, ${ }^{39,40}$ and normal cerebrospinal fluid inflammatory markers such as $\beta 2$ microglobulin. However, it appears that neopterin, a neuroinflammatory cerebrospinal fluid marker, remains abnormally elevated in patients with and without HAND on cART. ${ }^{41}$ The significance of low levels of HIV RNA replication $(<50$ copies $/ \mathrm{mL})$ is evolving, with some evidence that it may be partly responsible for the persistence of HAND. ${ }^{42,43}$ Longer HIV duration should be considered more carefully as it is becoming a more reliable marker of HAND, although its exact significance needs to be better determined, particularly in regards to patients' age. ${ }^{44}$ The reader should refer to other articles of this special issue in relation to newer biomarkers, comorbid conditions that may compound HAND development, including host genetic factors.

\section{Aging, cardiovascular disease (CVD), and renal dysfunction}

Several studies have shown increased cognitive impairment in older $\mathrm{HIV}+$ individuals receiving cART compared to younger HIV+ individuals receiving cART. ${ }^{45-47}$ There is currently no neuropsychological evidence that age and HIV interact substantially to produce a more severe neurocognitive profile in HAND. ${ }^{48}$ However, emerging data from neuroimaging studies, such as functional magnetic resonance imaging $^{49}$ and magnetic resonance spectroscopy (MRS $)^{50}$ have found that HIV-related brain injury is compounded in the oldest HIV+ individuals. This may represent a delay between the start of HIV-related brain injury and its eventual clinical expression as detected by neuropsychological assessment. Furthermore, there may be a survivor bias against finding a significant compounding effect of age in current patients who have survived through the pre-cART era, given that HAD was a risk factor for death. ${ }^{51}$ Thus older patients (age 60+ years) should receive more regular screening than younger individuals for HAND detection and progression. It should also be remembered that these patients are reaching an age when non-HIV-related neurodegenerative dementias become more prevalent. There is some evidence that this will lead to an increased burden of HIV+ patients with neurocognitive dysfunction. ${ }^{52}$

A significant body of evidence shows an increase in the risk of CVD in middle-aged HIV+ individuals as compared to their age-matched HIV-negative counterparts, even when corrected for CVD risk factors. ${ }^{53}$ Indeed, HIV+ individuals have a high rate of behavioral and demographic CVD risk factors. In the largest study conducted so far ( $\mathrm{n}=2945$; mean age 40 years), ${ }^{54}$ the prevalence of CVD risk factors was: smoking $43.3 \%$, diabetes $8.9 \%$, hypertension requiring medication $23.4 \%$, hypercholesterolemia requiring medication $25.1 \%$, and prior stroke $1.7 \%$. The reasons for a higher rate of CVD are thought to be related to the combined effect of HIV and cART (especially protease inhibitors, although these results are still debated) through HIV-related inflammation, hypercholesterolemia, elevated levels of very lowdensity cholesterol and lowered high-density lipoprotein cholesterol levels, ${ }^{55}$ raised triglycerides, visceral adiposity, and insulin resistance. ${ }^{53}$ Furthermore, CVD is a leading cause of dementia in the older general population and an increased risk factor for early AD. ${ }^{56}$ There is emerging evidence in neuropsychological studies that CVD is a new contributing factor to HAND. ${ }^{54,57,58}$ Our HIV and aging research program also has preliminary evidence that CVD affects regions of the brain that are associated with HIV-related brain injury (ie, caudate) as well as regions that have been associated with pathological aging (ie, posterior cingulate cortex).$^{59}$ Thus, active assessment and treatment of CVD is recommended, especially if the patient has a current or past history of HAND.

Renal dysfunction, a common comorbidity in HIVinfected patients, particularly those who are older, ${ }^{60}$ has not yet been assessed as a potential contributor to HAND in the cART era. However, recent studies in the general population have now linked chronic renal dysfunction with increased brain damage (including vascular brain damage) ${ }^{61}$ It is 
likely that chronic renal dysfunction will also emerge in the years to come as a new risk factor for HAND and a potential compounder of CVD-related brain injury in chronic HIV infection. Close monitoring of renal function and control of risk factors, especially hypertension, should be employed in HIV-infected patients, particularly those with HAND.

\section{Screening, detection, and long-term follow-up}

As mentioned above, standard neuropsychological assessment has become a key evaluation in HAND diagnosis. However, such evaluation is not always accessible as a priority. Also, it would be too costly from a public health perspective to examine all HIV+ patients for HAND. To this end, Cysique et $\mathrm{al}^{7}$ have suggested a staged approach, whereby individuals who are most at risk for HAND are assessed by a very brief screen, lasting only a few minutes. If deemed necessary, this can then be followed by a longer cognitive screen (approximately 15 minutes) as well as an assessment of mood and IADL (approximately 10 minutes). If the more formal cognitive screen is also positive then the patient should be referred for clinical assessment, neurological workup, and more extensive neuropsychological assessment where possible. To this aim, a neuro-algorithm, in the format of a very brief computerized screen, has been developed. It measures combined risk factors, with $78 \%$ accuracy for HAND classification. This publically available algorithm ${ }^{7}$ found that age, current CD4 cell count, past CNS HIV-related diseases, and current treatment duration were all predictors of HAND (as part of a weighted combination). Viral load suppression was not found to be a predictor. Because this algorithm was developed using a group with a restricted range of HIV disease severity, the authors are currently developing a new algorithm with patients who are more representative of primary care patients. Also, new risk factors, principally for CVD, were not taken into account in the first version of this algorithm. Other researchers are also building on this method to develop more comprehensive algorithms.

The early detection of HAND is becoming an important public health issue, especially as patients are aging and living with an increasing burden of comorbid conditions that are themselves risk factors for brain damage. Optimal methods for HAND detection at the primary care level are being assessed, but there are difficulties. As explained above, neurocognitive screening tools that are too brief will miss mild forms of HAND, especially in patients with high premorbid functioning. Indeed, while the HIV Dementia Scale ${ }^{62}$ may provide some information in moderate to severe cases it lacks sensitivity to milder forms of HAND. ${ }^{63}$ In general, for cognitive screens to be sensitive to mild HAND, they should at least assess psychomotor speed, verbal learning, and memory. ${ }^{64,65}$ Additionally, such screens must have corrections for demographic factors, and at least for age, education, and sex.

Lastly, screening tools for the long-term monitoring of $\mathrm{HIV}+$ individuals are under construction. Such tools need to reliably classify impairment by taking into account practice effects but also reliably indicate cognitive decline, as well as being able to monitor the cognitive benefit of long-term highly active antiretroviral therapy or any forthcoming adjunctive therapy.

\section{Current therapeutic options}

As yet, no adjunctive or neuroprotective therapies have effectively produced a clinically relevant level of benefit for patients with HAND. The search for novel pathogenic mechanisms and therapeutic approaches is under way. ${ }^{66}$

\section{NeurocART}

Some antiretrovirals are known to have better CNS penetration. To this effect, a score has been developed to systematically quantify its magnitude (the CNS penetration effectiveness score, or CPE) ${ }^{67} \mathrm{~A}$ cART regimen with good CNS penetration $(\mathrm{CPE}>7)$ has also been termed neurocART. ${ }^{68}$ Conclusive evidence of the superiority of neurocART at this point is lacking. Although retrospective studies ${ }^{69}$ showed no benefit, two European studies ${ }^{70,71}$ subsequently found systematic biases in the prescription of drugs with higher CPE in patients with HAD, some of whom may have been impaired for too long to fully recover. Results from observational prospective cohorts have been assessed qualitatively for rigor of method and quantitatively for power, and showed an average medium effect-size benefit for neurocART. ${ }^{68}$ Hopefully, the results of randomized clinical trials will definitively address the issue soon, but any trial has to be designed with at least adequate power and optimal neuropsychology methodology. ${ }^{68}$ In patients with HAND, or at risk for HAND, a neurocART regimen is recommended where possible (taking into consideration issues such as resistance, adherence, and adverse effects). Lastly, whether early versus delayed initiation of cART is beneficial on neurocognitive functions over-time is currently under investigation in a large-scale international study (Strategic Timing of AntiRetroviral Treatment [START] Neurology substudy), which will also assess the potential neurocART benefit as a second aim (see details at http://insight.ccbr.umn.edu/start/). 


\section{Long-term CART: direct and indirect neurotoxicities}

Despite abundant evidence that nucleoside reverse transcriptase inhibitors can disturb mitochondrial function ${ }^{72}$ and protease inhibitors proteosomal function, ${ }^{73}$ leading to neuropathy and lipodystrophy, there is only emerging evidence of CNS toxicity from antiretrovirals (ARVs). An MRS study showed that didanosine and/or stavudine use was associated with a significant decrease in concentrations of frontal white matter $\mathrm{N}$-acetyl aspartate (NAA, a marker of neuronal functioning). ${ }^{74}$ NAA depletion may reflect mitochondrial dysfunction and/or depletion of cellular respiration. However, MRS of the cerebellum was not reported, yet low NAA in the cerebellum has been found in $93 \%$ of patients with inherited mitochondrial diseases, ${ }^{75}$ a logically putative model for possible ARV CNS mitochondrial toxicity. Another tentative finding comes from the ACTG 5170 multicenter prospective study, ${ }^{76}$ where patients coming off cART improved neuropsychologically. Further, Pfeffer et $\mathrm{al}^{77}$ reported three cases of chronic progressive external ophthalmoplegia, a form of mitochondrial disorder that appeared to be associated with ARVs.

Vascular cognitive impairment is related to CNS vascular disease. Although controversial, particular ARVs have been associated with CVD (didanosine, abacavir, lopinavir, and indinavir) ${ }^{78,79}$ Ironically, these ARVs (with the exception of didanosine) are the very drugs that seem to be more efficacious in the brain. Evidence for CVD-related brain injury as a component of HAND in the cART era is emerging. ${ }^{80}$ Whether this is linked to some specific ARV is under investigation.

\section{Improvement in the assessment of everyday life functioning and cognitive recommendations for patients with HAND Better assessments of IADL}

Recent advances in neuropsychology research have seen the development of innovative strategies to better characterize and quantify IADL established upon performance-based measures that are adapted to HAND, even in its milder form. ${ }^{8,81-83}$ For example, in a recent study it was found that functionally impaired patients (as assessed formally) were more likely to be unemployed and more immunosuppressed, whereas those classified as functionally impaired from selfreport had more depressive symptoms. ${ }^{84}$ This empirically confirms previous findings ${ }^{26}$ and emphasizes the importance of classifying IADL reliably to determine ANI versus
MND prevalence correctly. ${ }^{85}$ These performance-based IADL measures are usually relatively lengthy and known to neuropsychologists and occupational therapists (see Heaton et $\mathrm{al}^{8}$ for original examples in HIV patients), but less well known to medical doctors. These batteries involve the completion of various artificial tasks that represent everyday activities, such as managing finances, medication management, ${ }^{81,86}$ and laboratory-based driving simulation ${ }^{83}$ (although the latter may be useful only in persons who are still driving and when public transport is not an option). Their use improves the classification of ANI and MND. ${ }^{84,85}$

Nevertheless, some of these tasks may still not be sensitive to the mildest form of HAND. An innovative research strategy was designed by Scott et a ${ }^{82}$ investigating one of the most demanding aspects of everyday life: multitasking. The authors compared demographically matched HIV-negative controls and HIV+ individuals on a comprehensive neuropsychological battery, questionnaires assessing mood and everyday functioning, and a novel standardized test of multitasking, which involved balancing the demands of four interconnected performance-based functional tasks (ie, financial management, cooking, medication management, and telephone communication). Multivariate prediction models revealed that multitasking deficits were uniquely predictive of IADL dependence beyond the effects of depression and global neurocognitive impairment, with excellent sensitivity (86\%) but modest specificity (57\%). While this task remains relatively lengthy, this is a potential option, if refined and shortened, as part of future quantitative screening.

Another strategy that has been extensively researched by Woods et $\mathrm{al}^{87}$ in HIV infection is the assessment of prospective memory. "Prospective memory is an innovative cognitive construct describing one's ability to remember to do something at a later time. ${ }^{\circ 8}$ Prospective memory is more closely associated with performance at the everyday life level than other standard cognitive domains. There is cumulative empirical evidence that it is reliably associated with medication adherence, more so than traditional assessment of cognitive functions ${ }^{89}$ Prospective memory has been shown to have increased ecological validity in patients with HAND and older patients with HAND. ${ }^{90}$ However, there is no short form of these assessments, and so their development as potential screens is still under research.

An alternative option may be to rely on both informantreported IADL and self-reported IADL. Indeed, this has long been used in the area of older-age dementia. This may help to eliminate inherent risks associated with the use of a self-report format (such as self-report bias), which is quite commonly used in HIV research. ${ }^{91}$ Self-report bias may lead to 
minimization when the patient is not aware of his/her deficits (which is relatively common in striatofrontal types of disorder such as HAND) or overreporting, which is linked more to depressive complaints than objective neuropsychological performance. ${ }^{83}$ Several strategies to reduce self-report bias have been proposed, including the possibility of utilizing both the individual's performance on measures of executive functioning and a thorough assessment of mood status ${ }^{26}$ to better interpret IADL complaints. ${ }^{92}$ The simple strategy of having an informant IADL would need to be innovatively validated and adapted to HIV research, because in many instances carers or partners are absent from attending HIV clinics. This may be due to various reasons, including the fact that HIV remains a relatively stigmatized illness and the significant other may be unwilling to come to the clinic, or that the patients themselves prefer to come alone. Most likely though, is the fact that the majority of HIV+ individuals are still relatively young (20-50 years old), ambulatory, and typically attend medical appointments alone. Future research should also aim to examine the opinion of a close friend, relative, or partner as to whether there have been changes in IADL. In clinical practice and if possible, both self-report and an informant IADL assessments are recommended.

\section{Recommendations to provide to the patient with HAND or at risk for HAND}

There are no empirically validated cognitive rehabilitation strategies for those with HAND. This is despite evidence of some benefit from use of such strategies in other forms of mild cognitive impairment. ${ }^{93}$ Nonetheless, a recent publication by Weber et $\mathrm{a} \mathrm{l}^{94}$ found that a self-generation strategy improved verbal memory recall in patients with HIV infection. More specifically, individuals performed better on measures of verbal memory when encoding was facilitated by an active self-generation of paired words rather than simple encoding by reading words aloud.

In the meantime, a psychosocial framework for improved everyday life and quality of life can be used in standard care that aims to maintain, and hopefully improve, cognitive reserve by taking action to prevent or partially alleviate cognitive loss/decline (see Vance and Struzick ${ }^{95}$ for details).

Additionally, as part of a standard neuropsychological assessment, the clinical neuropsychologist provides feedback to the patient, highlighting their profile of cognitive strengths as well as weaknesses, and how these may be used to function better in everyday life. Therefore, in addition to providing information pertaining to the patient's cognitive status, the neuropsychological assessment is also an initial step in a series of investigations from which the patient and their clinician will become cognizant of the difficulties and how to overcome some of them. For instance, the neuropsychologist may suggest various compensatory strategies. For example, for a patient who has difficulties learning and recalling verbal information but is adept with visual information, it may be recommended that information be presented to them in the form of pictures or graphs, and that they write down important points from discussion or have the information provided to them in writing. For a patient with slowing of cognition, the recommendations could include providing the individual with information in a paced manner and encouraging the individual to work at their own pace, avoiding rushing and time pressures. Importantly, such strategies are tailored to the individual's needs and to their specific cognitive profile as identified from assessment.

If the patient is unlikely to have the cognitive capacity to compensate for a deficit the neuropsychologist may suggest various external prompts and cues. For example, utilizing mobile phone reminders may help to compensate for difficulties with prospective memory (eg, remembering appointments near the appropriate time), while taking a notepad to meetings and appointments can help to reduce the demand on working memory (the amount of information they can mentally hold and consider at any one time). Importantly, the use of such strategies can assist an individual in maintaining an optimal level of independence, when they may otherwise have had to reduce their level of activity or rely on the help of a carer, and may also help to reduce stress.

The neuropsychological assessment has a number of functions: identifying the degree and nature of impairments, monitoring progress over time and response to treatments, and helping to identify those individuals who would benefit from social supports, brokered care, case management, or supported accommodation. Such services might be deemed necessary where the person is unable to independently manage their IADL, where they have difficulty maintaining adequate hygiene, adhering to their medication regime, meeting their dietary requirements, and keeping their living environment safe and free from hazards.

\section{Conclusions}

Clinical, research, and rehabilitation strategies are needed to better capture HAND long-term course, and these should lead to a revision of the current HAND (2007) diagnostic criteria. Briefer and more sensitive instruments to assess functional impairment objectively are needed, to improve the HAND criteria. New laboratory markers for chronic HAND are also needed. Finally, early detection remains important to 
capture prevalence/incidence reliably, adequately plan future public health policies, and adequately plan clinical care over the long term including preventive care for comorbid factors associated with aging.

\section{Acknowledgments}

This review is dedicated to our patients and our HIV+ and HIV- research participants. We thank them for their time in our research studies as well as their insight, comments, and constructive criticisms.

\section{Disclosure}

BJ Brew has received: speaking honoraria from Viiv, Boehringer Ingelheim, Abbott, Merck Serono, and Gilead; advisory board fees from Biogen Idec, Viiv and Abbott; royalty fees from Oxford University Press and Cambridge University Press; grant support from Biogen Idec, Neurogesx, Viiv and Merck Serono as well as National Institutes of Health and the National Health and Medical Research Council of Australia. Lucette A. Cysique has received: speaking honoraria from Viiv, Abbott and grant support from Abbott and Merck Serono as well as the National Health and Medical Research Council of Australia and the University of New South Wales in Australia. Dr. Cysique is also an academic consultant for the Canadian Trial Network and for CogState, Ltd.

The other authors report no conflict of interest

\section{References}

1. Cysique L, Maruff P, Brew B. Prevalence and pattern of neuropsychological impairment in human immunodeficiency virus-infected/acquired immunodeficiency syndrome (HIV/AIDS) patients across pre- and post-highly active antiretroviral therapy eras: a combined study of two cohorts. J Neurovirol. 2004;10:350-357.

2. Brew BJ. HIV Neurology (Contemporary Neurology Series). Oxford: Oxford University Press; 2001:53-90.

3. White DA, Heaton R, Monsch AU. Neuropsychological studies of asymptomatic human immunodeficiency virus-type 1 infected individuals. $J$ Int Neuropsychol Soc. 1995;1:304-315.

4. Scott JC, Woods SP, Carey CL, Weber E, Bondi MW, Grant I. Neurocognitive consequences of HIV infection in older adults: an evaluation of the "cortical" hypothesis. AIDS Behav. 2011;15(6):1187-1196.

5. McArthur JC, Haughey N, Gartner S, et al. Human immunodeficiency virus-associated dementia: an evolving disease. J Neurovirol. 2003;9:205-221.

6. Heaton RK, Franklin DR, Ellis RJ, et al. HIV-associated neurocognitive disorders before and during the era of combination antiretroviral therapy: differences in rates, nature, and predictors. J Neurovirol. 2010;17(1):3-16.

7. Cysique LA, Murray JM, Dunbar M, Jeyakumar V, Brew BJ. A screening algorithm for HIV-associated neurocognitive disorders. HIV Med. 2010;11(10):642.

8. Heaton RK, Marcotte TD, Mindt MR, et al. The impact of HIV-associated neuropsychological impairment on everyday functioning. J Int Neuropsychol Soc. 2004;10(3):317-331.

9. Ettenhofer ML, Foley J, Castellon SA, Hinkin CH. Reciprocal prediction of medication adherence and neurocognition in HIV/AIDS. Neurology. 2010;74(15):1217-1222.
10. Gorman AA, Foley JM, Ettenhofer ML, Hinkin CH, van Gorp WG Functional consequences of HIV-associated neuropsychological impairment. Neuropsychol Rev. 2009;19(2):186-203.

11. Tozzi V, Balestra P, Bellagamba R, et al. Persistence of neuropsychologic deficits despite long-term highly active antiretroviral therapy in patients with HIV-related neurocognitive impairment: prevalence and risk factors. J Acquir Immune Defic Syndr. 2007;45(2):174-182.

12. Cysique LA, Maruff P, Brew BJ. Variable benefit in neuropsychological function in HIV-infected HAART-treated patients. Neurology. 2006;66(9):1447-1450.

13. Heaton RK, Clifford DB, Franklin DR Jr, et al. HIV-associated neurocognitive disorders persist in the era of potent antiretroviral therapy: CHARTER Study. Neurology. 2010;75(23):2087-2096.

14. Heaton RK, Franklin DR, Ellis RJ, et al. HIV-associated neurocognitive disorders before and during the era of combination antiretroviral therapy: differences in rates, nature, and predictors. J Neurovirol. 2011;17(1): $3-16$.

15. Sacktor N, McDermott M, Marder K, et al. HIV-associated cognitive impairment before and after the advent of combination therapy. J Neurovirol. 2002;8:136-142.

16. Cysique LA, Brew BJ. Prevalence of non-confounded HIV-associated neurocognitive impairment in the context of undetectable plasma viral load. J Neurovirol. 2011;17(2):176-183.

17. Robertson KR, Smurzynski M, Parsons TD, et al. The prevalence and incidence of neurocognitive impairment in the HAART era. AIDS. 2007 21(14):1915-1921.

18. Garvey L, Surendrakumar V, Winston A. Low rates of neurocognitive impairment are observed in neuro-asymptomatic HIV-infected subjects on effective antiretroviral therapy. HIV Clin Trials. 2011;12(6): 333-338.

19. Simioni S, Cavassini M, Annoni JM, et al. Cognitive dysfunction in HIV patients despite long-standing suppression of viremia. AIDS. 2010; 24(9):1243-1250.

20. Antinori A, Arendt G, Becker JT, et al. Updated research nosology for HIV-associated neurocognitive disorders. Neurology. 2007;69(18): 1789-1799.

21. American Academy of Neurology, Dana Consortium. Clinical confirmation of the American Academy of Neurology algorithm for HIV-1-associated cognitive/motor disorder (Dana Consortium on therapy for HIV dementia and related cognitive disorders). Neurology. 1996;47:1247-1253.

22. Janssen R, Saykin A, Canon L, et al. Neurological and neuropsychological manifestations of HIV-1 infection: association with AIDS-related complex but not asymptomatic HIV-1 infection. Ann Neurol. 1989(26): 592-600.

23. Janssen R, Comblath D, Hopkins J, et al. Nomenclature and research case definitions for neurologic manifestations immunodeficiency virus type-1 (HIV-1): reports of a working group of the American Academy of Neurology AIDS Task Force. Neurology. 1991;41:778-785.

24. McKhann G, Drachman D, Folstein M, Katzman R, Price D, Stadlan EM. Clinical diagnosis of Alzheimer's disease: report of the NINCDS-ADRDA Work Group under the auspices of Department of Health and Human Services Task Force on Alzheimer's Disease. Neurology. 1984;34(7):939-944.

25. Chelune G, Heaton R, Lehman R. Neuropsychological and personality correlates of patient's complaints of disability. In: Tarter R, Goldstein G, editors. Advances in Clinical Neuropsychology. New York: Plenum Press; 1986:95-126.

26. Cysique LA, Deutsch R, Atkinson JH, et al. Incident major depression does not affect neuropsychological functioning in HIV-infected men. $J$ Int Neuropsychol Soc. 2007;13(1):1-11.

27. Goodkin K, Wilkie FL, Concha M, et al. Aging and neuro-AIDS conditions and the changing spectrum of HIV-1-associated morbidity and mortality. J Clin Epidemiol. 2001;54 Suppl 1:S35-S43.

28. Clarke DE, van Reekum R, Simard M, et al. Apathy in dementia: clinical and sociodemographic correlates. JNeuropsychiatry Clin Neurosci. 2008;20(3):337-347. 
29. Lane TA, Moore D, Batchelor J, Brew BJ, Cysique LA. Facial emotional processing in HIV infection: relation to neurocognitive and neuropsychiatric status. Neuropsychology. 2012. In press.

30. Gisslen M, Price RW, Nilsson S. The definition of HIV-associated neurocognitive disorders: are we overestimating the real prevalence? BMC Infect Dis. 2011;11:356.

31. Cherner M, Cysique L, Heaton RK, et al. Neuropathologic confirmation of definitional criteria for human immunodeficiency virus-associated neurocognitive disorders. J Neurovirol. 2007;13(1):23-28.

32. Heaton RK, Franklin D, Woods S, et al. Asymptomatic Mild HIVAssociated Neurocognitive Disorder Increases Risk for Future Symptomatic Decline: A CHARTER Longitudinal Study. Paper presented at Conference on Retroviruses and Opportunistic Infections; March 5-8, 2012; Seattle, WA.

33. Stern Y, McDermott M, Albert S, et al. Factors associated with incident human immunodefiency virus-dementia. Arch Neurol. 2001;58:473-479.

34. Heaton RK, Miller SW, Taylor MJ, Grant I. Revised Comprehensive Norms for an Expanded Halstead-Reitan Battery: Demographically Adjusted Neuropsychological Norms for African American and Caucasian Adults Scoring Program. Odessa: Psychological Assessment Resources; 2004.

35. Ingraham L, Aiken C. An empirical approach to determining criteria for abnormality in test batteries with multiple measures. Neuropsychology. 1996;10:120-124.

36. Cysique LA, Brew BJ. Neuropsychological functioning and antiretroviral treatment in HIV/AIDS: a review. Neuropsychol Rev. 2009;19(2): 169-185.

37. Ellis RJ, Badiee J, Vaida F, et al. CD4 nadir is a predictor of HIV neurocognitive impairment in the era of combination antiretroviral therapy. AIDS. 2011;25(14):1747-1751.

38. Valcour V, Yee P, Williams AE, et al. Lowest ever CD4 lymphocyte count (CD4 nadir) as a predictor of current cognitive and neurological status in human immunodeficiency virus type 1 infection - the Hawaii Aging with HIV Cohort. J Neurovirol. 2006;12(5):387-391.

39. Cysique LA, Brew BJ, Halman M, et al. Undetectable cerebrospinal fluid HIV RNA and beta-2 microglobulin do not indicate inactive AIDS dementia complex in highly active antiretroviral therapy-treated patients. J Acquir Immune Defic Syndr. 2005;39(4):426-429.

40. Sevigny JJ, Albert SM, McDermott MP, et al. Evaluation of HIV RNA and markers of immune activation as predictors of HIV-associated dementia. Neurology. 2004;63:2084-2090.

41. Eden A, Fuchs D, Hagberg L, et al. HIV-1 viral escape in cerebrospinal fluid of subjects on suppressive antiretroviral treatment. $J$ Infect Dis. 2010;202(12):1819-1825.

42. Clements JE, Gama L, Graham DR, Mankowski JL, Zink MC. A simian immunodeficiency virus macaque model of highly active antiretroviral treatment: viral latency in the periphery and the central nervous system. Curr Opin HIV AIDS. 2011;6(1):37-42.

43. Schnell G, Joseph S, Spudich S, Price RW, Swanstrom R. HIV-1 replication in the central nervous system occurs in two distinct cell types. PLoS Pathog. 2011;7(10):e1002286.

44. Bhaskaran K, Mussini C, Antinori A, et al. Changes in the incidence and predictors of human immunodeficiency virus-associated dementia in the era of highly active antiretroviral therapy. Ann Neurol. 2008;63(2): 213-221.

45. Becker JT, Lopez OL, Dew MA, Aizenstein HJ. Prevalence of cognitive disorders differs as a function of age in HIV virus infection. AIDS. 2004;18 Suppl 1:S11-S18.

46. Sacktor N, Skolasky R, Selnes OA, et al. Neuropsychological test profile differences between young and old human immunodeficiency virus-positive individuals. J Neurovirol. 2007;13(3):203-209.

47. Valcour V, Shikuma C, Watters M, et al. Higher frequency of dementia in older HIV-1 individuals. The Hawaii aging with HIV-1 cohort. Neurology. 2004;63:822-827.

48. Cysique LA, Maruff P, Bain MP, Wright E, Brew BJ. HIV and age do not substantially interact in HIV-associated neurocognitive impairment. J Neuropsychiatry Clin Neurosci. 2011;23(1):83-89.
49. Ances BM, Vaida F, Yeh MJ, et al. HIV infection and aging independently affect brain function as measured by functional magnetic resonance imaging. J Infect Dis. 2010;201(3):336-340.

50. Chang L, Wong V, Nakama $\mathrm{H}$, et al. Greater than age-related changes in brain diffusion of HIV patients after 1 year. J Neuroimmune Pharmacol. 2008;3(4):265-274.

51. Mayeux R, Stern Y, Tang MX, et al. Mortality risks in gay men with human immunodeficiency virus infection and cognitive impairment. Neurology. 1993;43(1):176-182.

52. Cysique LA, Bain MP, Brew BJ, Murray JM. The burden of HIVassociated neurocognitive impairment in Australia and its estimates for the future. Sex Health. 2011;8(4):541-550.

53. Carr A. Pathogenesis of cardiovascular disease in HIV infection. Curr Opin HIV AIDS. 2008;3(3):234-239.

54. Wright EJ, Grund B, Robertson K, et al. Cardiovascular risk factors associated with lower baseline cognitive performance in HIV-positive persons. Neurology. 2010;75(10):864-873.

55. Das S. Risk of cardiovascular disease in HIV-infected patients. J Antimicrob Chemother. 2009;65(3):386-389.

56. Gorelick PB, Scuteri A, Black SE, et al. Vascular contributions to cognitive impairment and dementia: a statement for healthcare professionals from the American Heart Association/American Stroke Association. Stroke. 2011;42(9):2672-2713.

57. Becker JT, Kingsley L, Mullen J, et al. Vascular risk factors, HIV serostatus, and cognitive dysfunction in gay and bisexual men. Neurology. 2009;73(16):1292-1299.

58. Valcour VG, Sacktor NC, Paul RH, et al. Insulin resistance is associated with cognition among HIV-1-infected patients: the Hawaii Aging with HIV cohort. J Acquir Immune Defic Syndr. 2006;43(4): 405-410.

59. Cysique LA. Evidence of Early Cardiovascular-Related Metabolic Brain Injury in Older HIV+ Individuals Using Single Voxel Magnetic Resonance Spectroscopy. Paper presented at: Fourth International Meeting on HIV Infection and the Central Nervous System; July 15-16, 2011; Rome, Italy.

60. Post FA, Holt SG. Recent developments in HIV and the kidney. Curr Opin Infect Dis. 2009;22(1):43-48.

61. Ryu WS, Lee SH, Kim CK, Kim BJ, Yoon BW. The relation between chronic kidney disease and cerebral microbleeds: difference between patients with and without diabetes. Int J Stroke. 2012;2012(16): $1747-4949$.

62. Davis H, Skolasky RJ, Selnes O, Burgess D, McArthur J. Assessing HIV-associated dementia: modified HIV dementia scale versus the Grooved Pegboard. AIDS Read. 2002;12(1):32-33.

63. Bottiggi KA, Chang JJ, Schmitt FA, et al. The HIV Dementia Scale: predictive power in mild dementia and HAART. J Neurol Sci. 2007; 260(1-2):11-15.

64. Cysique L, Maruff P, Brew B. The neuropsychological profile of symptomatic, AIDS and ADC patients in the pre-HAART era: a metaanalysis. J Int Neuropsychol Soc. 2006;12:1-15.

65. Carey CL, Woods SP, Rippeth JD, et al. Initial validation of a screening battery for the detection of HIV-associated cognitive impairment. Clin Neuropsychol. 2004;18(2):234-248.

66. Kaul M. HIV-1 associated dementia: update on pathological mechanisms and therapeutic approaches. Curr Opin Neurol. 2009;22(3):315-320.

67. Letendre S, Marquie-Beck J, Capparelli E, et al. Validation of the CNS Penetration-Effectiveness rank for quantifying antiretroviral penetration into the central nervous system. Arch Neurol. 2008;65(1):65-70.

68. Cysique LA, Waters EK, Brew BJ. Central nervous system antiretroviral efficacy in HIV infection: a qualitative and quantitative review and implications for future research. BMC Neurol. 2011;11(148):148.

69. McManus H, Li PC, Nolan D, et al. Does use of antiretroviral therapy regimens with high central nervous system penetration improve survival in HIV-infected adults? HIV Med. 2011;12(10):610-619.

70. Garvey L, Winston A, Walsh J, et al. Antiretroviral therapy CNS penetration and HIV-1-associated CNS disease. Neurology. 2011;76(8): 693-700. 
71. Lanoy E, Guiguet M, Bentata M, et al. Survival after neuroAIDS: association with antiretroviral CNS Penetration-Effectiveness score Neurology. 2011;76(7):644-651.

72. Kohler JJ, Lewis W. A brief overview of mechanisms of mitochondrial toxicity from NRTIs. Environ Mol Mutagen. 2007;48(3-4):166-172.

73. Piccinini M, Rinaudo MT, Anselmino A, et al. The HIV protease inhibitors nelfinavir and saquinavir, but not a variety of HIV reverse transcriptase inhibitors, adversely affect human proteasome function. Antivir Ther. 2005;10(2):215-223.

74. Schweinsburg BC, Taylor MJ, Alhassoon OM, et al. Brain mitochondrial injury in human immunodeficiency virus-seropositive (HIV+) individuals taking nucleoside reverse transcriptase inhibitors. J Neurovirol. 2005;11(4):356-364.

75. Saneto RP, Friedman SD, Shaw DW. Neuroimaging of mitochondrial disease. Mitochondrion. 2008;8(5-6):396-413.

76. Robertson KR, Su Z, Margolis DM, et al. Neurocognitive effects of treatment interruption in stable HIV-positive patients in an observational cohort. Neurology. 2011;74(16):1260-1266.

77. Pfeffer G, Cote HC, Montaner JS, Li CC, Jitratkosol M, Mezei MM. Ophthalmoplegia and ptosis: mitochondrial toxicity in patients receiving HIV therapy. Neurology. 2009;73(1):71-72.

78. Friis-Moller N, Thiebaut R, Reiss P, et al. Predicting the risk of cardiovascular disease in HIV-infected patients: the data collection on adverse effects of anti-HIV drugs study. Eur J Cardiovasc Prev Rehabil. 2010;17(5):491-501.

79. Law MG, Friis-Moller N, El-Sadr WM, et al. The use of the Framingham equation to predict myocardial infarctions in HIV-infected patients: comparison with observed events in the D:A:D Study. HIV Med. 2006; 7(4):218-230.

80. Cruse B, Cysique LA, Markus R, Brew BJ. Cerebrovascular disease in HIV-infected individuals in the era of highly active antiretroviral therapy. J Neurovirol. 2012; in press.

81. Gandhi NS, Skolasky RL, Peters KB, et al. A comparison of performance-based measures of function in HIV-associated neurocognitive disorders. J Neurovirol. 2011;17(2):159-165.

82. Scott JC, Woods SP, Vigil O, et al. A neuropsychological investigation of multitasking in HIV infection: implications for everyday functioning. Neuropsychology. 2011;25(4):511-519.

83. Thames AD, Becker BW, Marcotte TD, et al. Depression, cognition, and self-appraisal of functional abilities in HIV: an examination of subjective appraisal versus objective performance. Clin Neuropsychol. $2011 ; 25(2): 224-243$.
84. Blackstone K, Moore DJ, Heaton RK, et al. Diagnosing symptomatic HIV-associated neurocognitive disorders: self-report versus performance-based assessment of everyday functioning. $J$ Int Neuropsychol Soc. 2012;18(1):79-88.

85. Foley JM, Wright MJ, Gooding AL, et al. Operationalization of the updated diagnostic algorithm for classifying HIV-related cognitive impairment and dementia. Int Psychogeriatr. 2010;19:1-9.

86. Thames AD, Kim MS, Becker BW, et al. Medication and finance management among HIV-infected adults: the impact of age and cognition. J Clin Exp Neuropsychol. 2011;33(2):200-209.

87. Woods SP, Dawson MS, Weber E, Gibson S, Grant I, Atkinson JH Timing is everything: antiretroviral nonadherence is associated with impairment in time-based prospective memory. J Int Neuropsychol Soc. 2009; 15(1):42-52.

88. Zogg JB, Woods SP, Sauceda JA, Wiebe JS, Simoni JM. The role of prospective memory in medication adherence: a review of an emerging literature. J Behav Med. 2011;2011:13.

89. Woods SP, Iudicello JE, Moran LM, Carey CL, Dawson MS, Grant I. $\mathrm{HIV}$-associated prospective memory impairment increases risk of dependence in everyday functioning. Neuropsychology. 2008;22(1): $110-117$.

90. Weber E, Woods SP, Delano-Wood L, Bondi MW, Gilbert PE, Grant I. An examination of the age-prospective memory paradox in HIV-infected adults. J Clin Exp Neuropsychol. 2011;33(10):1108-1118.

91. Hinkin $\mathrm{CH}$, van Gorp WG, Satz P, et al. Actual versus self-reported cognitive dysfunction in HIV-1 infection: memory-metamemory dissociations. J Clin Exp Neuropsychol. 1996;18(3):431-443.

92. Rourke SB, Halman MH, Bassel C. Neurocognitive complaints in HIV-infection and their relationship to depressive symptoms and neuropsychological functioning. J Clin Exp Neuropsychol. 1999;21(6): 737-756.

93. Martin M, Clare L, Altgassen AM, Cameron MH, Zehnder F. Cognition-based interventions for healthy older people and people with mild cognitive impairment. Cochrane Database Syst Rev. 2011;19(1): CD006220.

94. Weber E, Woods SP, Kellogg E, Grant I, Basso MR. Self-generation enhances verbal recall in individuals infected with HIV.J Int Neuropsychol Soc. 2012;18(1):128-133.

95. Vance DE, Struzick TC. Addressing risk factors of cognitive impairment in adults aging with HIV: a social work model. J Gerontol Soc Work. 2007;49(4):51-77.
Neurobehavioral HIV Medicine

\section{Publish your work in this journal}

Neurobehavioral HIV Medicine is an international, peer-reviewed, open access journal focusing on advances in research in HIV/ AIDS, with specific reference to the neurological, psychiatric and behavioral consequences of the disease, concomitant infections and specific antiretroviral therapy. The manuscript

\section{Dovepress}

management system is completely online and includes a very quick and fair peer-review system, which is all easy to use Visit http://www.dovepress.com/testimonials.php to read real quotes from published authors. 